\title{
Signaling Flux Redistribution concept can switch survival to apoptosis in cancer cells
}

\author{
Daiki Yamada, Kentaro Hayashi, Vincent Piras, Masaru Tomita, Masa Tsuchiya and Kumar Selvarajoo
}

The tumor necrosis factor related apoptosis-inducing ligand (TRAIL) induces apoptosis in certain types of carcinoma. However, in majority of malignant tumors, it has no effect. Here we analyzed cell survival and apoptotic pathways triggered by TRAIL. A computational model based on perturbation-response approach with mass-action equations was developed. All 48 parameter values of the model were selected by genetic algorithm that minimizes deviation between experimental and simulation data for human fibrosarcoma cells. Subsequently, we show in silico knock-down of a cell survival pathway molecule could potentially enhance the activation of apoptosis through Signaling Flux Redistribution (SFR).

Keywords - TRAIL, perturbation-response, apoptosis, cancer, signaling flux redistribution.

\section{Purpose}

$\mathrm{T}$ HE intensely investigated anti-cancer treatments have focused on the development of strategies that are both non-invasive and non-toxic. The conventional chemotherapy and radiotherapy remain suboptimal due to the large number of cancer deaths despite careful treatment. Over the last decade, the search to induce apoptosis (programmed cell death) in cancer cells have led to the emergence of a new and fast growing field termed cancer immunology. Here, the interactions between immune system with malignant cancers have shown the suppression of the disease progression [1].

Among the many immune factors, the TRAIL is known to induce cellular apoptosis [1]. Recently, TRAIL has received primal attention due to its ability to recognize and induce apoptosis of tumors and metastases while leaving normal cells unscathed. However, several cancers, such as melanoma and neuroblastoma, are resistant to TRAIL treatment [2].

\section{APPROACH}

To better understand the TRAIL resistance mechanisms which occur due to increased cell survival activity, we developed a computational model based on perturbation-response approach using the up-to date knowledge of TRAIL signaling topology. The approach utilizes the conservation law of signal propagation together with first-order response equations $[3,4]$. We also utilized genetic algorithm to choose the parameter values of the model, from multiple runs, for all 48 reactions that best fit

Acknowledgements: This work was supported by Japan Science and Technology Agency/Core Research for Evolutional Science and Technology (JST CREST) and the Yamagata Prefecture and Tsuruoka City, Japan.

Institute for Advanced Biosciences, Keio University, Tsuruoka, Japan. with time-course experimental activation profiles of cell survival (IאB, JNK, p38) and apoptotic (Caspases-8 and -3) molecules for human fibrosarcoma (HT1080) cells.

To verify parameter uniqueness of the wildtype (WT) model, another experimental condition (FADD knock-down (KD)) was used as a test. This time, however, the model did not recapitulate experimental outcome (data not shown). Using simple linear rules [3], we deciphered a novel FADD-independent pathway whose inclusion in the model resulted in improved simulations for both WT and FADD KD conditions (Fig. 1).
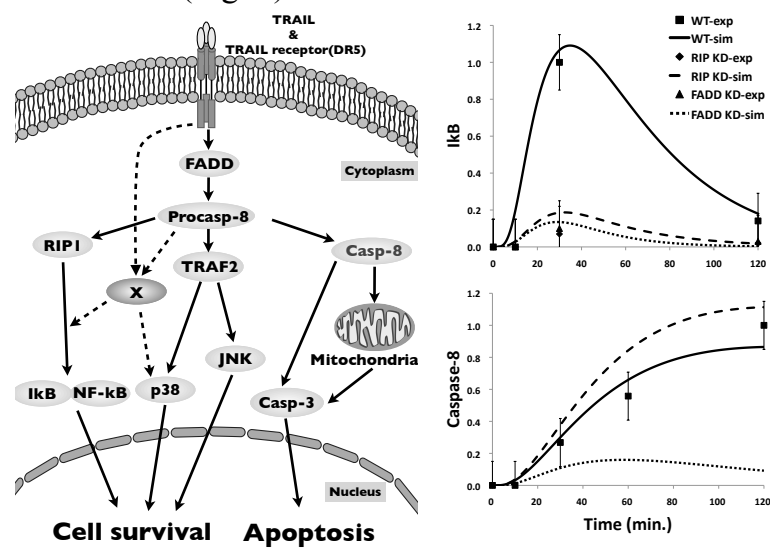

Fig. 1. Left panel: Simplified schematic of TRAIL signaling pathways reflecting novel predicted pathways (dotted lines with X). Right panels: Comparison of experimental and simulated IкB (top) and Caspase-8 (bottom) activation profiles using modified model in WT, FADD KD and RIP1 KD for HT1080 cells. Note: experimental data for caspase-8 activation is only available for wildtype. In this abstract, data is not shown for $\mathrm{p} 38$, JNK and caspase- 3 response profiles due to space restriction.

We used the updated model to overcome TRAIL resistance. By targeting the molecules at cell survival pathway junction, namely RIP1, apoptotic pathway is enhanced through SFR [4].

\section{CONCLUSION}

The presented approach shows that SFR could be used as a strategy to further uncover an optimal target, whose removal would produce a dual mode of cell survival pathway suppression and apoptosis enhancement. This might provide a valuable contribution towards sensitizing TRAIL-based cancer therapy.

\section{REFERENCES}

[1] Pennarun B, et al (2010) Playing the DISC: Turning on TRAIL death receptor-mediated apoptosis in cancer. Biochim Biophys Acta.1805, 123-140.

[2] Lidong Z, Bingliang F (2005) Mechanisms of resistance to TRAIL-induced apoptosis in cancer. Cancer Gene Therapy. 12, 228-237

[3] Selvarajoo K, Tomita M, Tsuchiya M (2009) Can complex cellular processes understood by simple linear rules? J Bioinform Comput Biol. 7, 243-268.

[4] Selvarajoo K, et al (2008) Signaling Flux Redistribution at Toll-like Receptor Pathway Junctions. PLoS ONE. 3, e3430. 